\title{
"Tooth and Truth": Brain Activation During Passive Listening to Concrete and Abstract Nouns
}

\author{
Weiss S. ${ }^{1}$, Mueller H.M. ${ }^{*}, 1,2$, Mertens M. ${ }^{3}$ and Woermann F.G. ${ }^{3}$ \\ ${ }^{I}$ CITEC Center of Excellence "Cognitive Interaction Technology", Bielefeld University, Germany \\ ${ }^{2}$ SFB 673, Experimental Neurolinguistics Group, Bielefeld University, Germany \\ ${ }^{3}$ MRI Unit, Bethel Epilepsy Center, Ev. Krankenhaus, Bielefeld, Germany
}

\begin{abstract}
The aim of this functional magnetic resonance imaging (fMRI) study was to investigate brain activation associated with the auditory perception of concrete and abstract German nouns during a passive listening task. This procedure allows us to investigate inherent linguistic properties common to either concrete or abstract concepts rather than cognitive operations due to the performance of a given specific task. In addition, a new baseline condition (pseudospeech) was used allowing us to monitor language-relevant activation common to both word types besides the pre-lexical analysis of the speech sound. Fixed effects analyses indicated increased activation of left hemispheric ventromedial prefrontal brain regions specifically responsive to the comprehension of concrete nouns by the current participants. This was interpreted in terms of the multisensory representation of concrete nouns comprising not only elicitation of visual images but also of multimodal sensoric and manipulation-related context. In contrast, abstract nouns did not activate any brain region exclusively. Random effects analyses revealed only very slight differences between concrete and abstract nouns in left ventromedial prefrontal brain activation. This suggests that the passive listening task is not suitable to canalize the higher cognitive variability in processing abstract items which seem to result in less coherent brain activation.
\end{abstract}

Keywords: Abstract, concrete, spoken language, pseudo-speech, lexicon, nouns, fMRI.

\section{INTRODUCTION}

Investigating the organization of the mental lexicon and its neurophysiological correlates is one of the most challenging topics in the cognitive neuroscience of language. It is not known, for example, if subclasses of nouns (e.g., "concrete $v s$. abstract") are artificial classes based on theoretical considerations in philosophy of language and linguistics or if they refer to real cognitive categories, which were established during the phylogenetic development of cognition and language. If the latter were the case, the questions would arise as to how these word classes are functionally represented within the brain and whether they are associated with the activity of similar or distinct neuronal networks.

From a linguistic point of view, concrete nouns (e.g., bottle) are characterized by high reference and high intension (association). They represent individual entities of the world having constant features, which can be referred to in the three-dimensional space. In contrast, abstract nouns (e.g., soul) have no spatio-temporal concreteness and their individuality is low [1]. During language acquisition children learn concrete nouns together with their cognitive-motoric referents before they learn to comprehend or produce abstract nouns or verbs [2]. Abstract concepts are acquired through their relationship to other concepts and their use in sentences. Thus, whereas concrete nouns refer to specific

*Address correspondence to this author at the SFB 673 - Exp. Neurolinguistics Group, Bielefeld University, P.O. Box 100131, 33501 Bielefeld, Germany; Tel: +49-521-106-5307; Fax: +49-521-106-6447; E-mail: horst.mueller@uni-bielefeld.de objects and may be represented not only in the visual but also in the auditory, tactile, olfactory and gustatory format, abstract linguistic stimuli refer to ideas and concepts, have no physical referents and are represented in a propositional format.

Indisputably, concreteness has a major influence on word recognition and comprehension. Numerous psycholinguistic studies investigating reaction time, lexical and semantic decision, priming, dichotic listening, divided visual field activation, free and cued recall, and sentence comprehension have demonstrated that concrete linguistic stimuli have many cognitive processing advantages over abstract linguistic stimuli [3-5]. This processing difference is also supp-orted by neuropsychological case studies showing selective difficulties in processing abstract nouns in deep dyslexia [6], deep dysphasia [7], and aphasia [8]. Most interestingly, the investigation of a patient with semantic refractory access dysphasia indicated that concrete concepts are represented in a categorical network and abstract ones in an associative network [9].

Recently, neuroimaging studies have been performed in order to address the question of the existence of a qualitative (activity in different systems) or a quantitative (different activity in a single system) difference in brain activity associated with the processing of concrete and abstract words. On the one hand, EEG studies support the notion of there being different neuronal systems involved [10-13]. On the other hand, there are indications for a common semantic system but a quantitative difference [14]. Holcomb et al. [15] postulated the so-called "context extended dual coding 
theory" suggesting a similar effect of context on both word types in a linguistic-based system and a larger influence of context on the concrete words in a separate image-based system. Further evidence for the partial involvement of different neuronal networks in the processing of concrete and abstract nouns came from hemodynamic studies using PET or fMRI. In these studies, processing abstract compared to concrete concepts showed more consistent results mainly activating three large brain regions. They have elicited higher activation of left frontal regions [13, 16-21], the left superior temporal cortex [13, 22-25] and large regions of the right superior, middle and inferior temporal cortex $[22,23$, 26]. In contrast, processing concrete concepts has yielded much more divergent results with the activation of more and distributed brain regions mainly in the left hemisphere. The most consistent activation has been found in the left inferior temporal/fusiform gyrus $[16,22-24,27,28]$. In addition, higher activation has occurred in large areas of the left frontal lobe [18, 22], occipital association cortex [23, 24, 29], left precentral [22, 24], and lower left parietal areas [18, $22,30]$ but also right hemispheric areas [13].

The rationale for the present fMRI-study was to investigate participants listening to auditorily presented concrete and abstract concepts as independently as possible from any common, intentional task or external instruction since it is not clear to which extend different tasks would influence the concreteness effect. Based on the behavioral and neurophysiological findings reported above we hypothesize that even without a given task, processing concrete compared to abstract nouns should elicit more activation in distributed brain regions, in particular secondary sensory, limbic and association cortex. Further, a baseline condition was designed in order to monitor higher-order linguistic processes common to both word types. Frequently, it has been shown that a resting condition itself is influenced by many uncontrollable factors [31] and thus is not an ideal baseline condition for monitoring activation of language-relevant brain structures. Therefore, so-called pseudo-speech stimuli were used, which are completely artificial "wordless" signals but comparable to real speech signals in complexity and length [32]. These signals should demonstrate language-relevant common activation, which is extinguished in the direct comparison of concrete and abstract nouns.

\section{MATERIAL AND METHODS}

\section{Participants}

Fourteen monolingual students ( $8 \mathrm{f}, 6 \mathrm{~m}$ ) aged 19-28 years (mean $23.7 \pm 3.5$ ) with German as their native language participated in the experiment. They all had intact audiograms of both ears and reported no history of any significant neurological or psychiatric illness. The participants anatomical data were formally screened for abnormalities by a neuroradiologist. Thirteen of the participants were right-handed, two of them had a tendency for left-handedness in childhood and one had left-handed relatives. Their laterality quotients on a modified version of the Edinburgh Handedness Inventory ranged from 84 to $100 \%$, indicating strong right-handed preference for all subjects. One participant was left-handed according to the questionnaire. However, a standardized verbal fluency test performed at the end of each fMRI-session exhibited left-lateralized language dominance for all participants [33].

\section{Stimuli}

Fifty concrete and fifty abstract disyllabic German nouns were selected and equated for frequency of occurrence according to the CELEX database [34]. Concrete nouns showed an average of 115.5 (SD 33.8) and abstract nouns 116,4 (SD 45.6) per million words. Words were matched on imageability and concrete-/abstractness according to studies of Baschek et al. [35] and Offe et al. [36]. On a bipolar scale $(-20<0<+20)$ the score for the concrete nouns was $16.5 \pm$ 0.75 for imagery and $16.2 \pm 2.4$ for concrete-/abstractness. The abstract nouns had $-3.18 \pm 4.22$ for imagery and $-4.76 \pm$ 4.38 for concrete-/abstractness. Concrete and abstract nouns differed significantly for the variables "imagery" and "concrete-/ abstractness" ( $t$-test, $2 p \leq 0.0001)$.

Nouns were spoken by a professional male German speaker and recorded in a professional recording studio (Bielefeld University). Stimuli were digitized at $41 \mathrm{kHz}, 16$ bit and had a mean articulatory duration of $652 \mathrm{~ms} \pm 87 \mathrm{~ms}$ (concrete nouns $\varnothing=603 \pm 83 \mathrm{~ms}$; abstract nouns $\varnothing=700 \pm$ $90 \mathrm{~ms}$ ). During the fMRI scans stimuli were presented binaurally via computer, HiFi-amplifier, paired plastic tubes, and customized closed headphones.

In a further condition participants perceived pseudospeech stimuli [32], which were created in order to provide a suitable baseline for only analyzing language-relevant processes associated with the comprehension of lexical items. For that purpose, an artificial complex signal was created by modulation of a carrier wave $(200 \mathrm{~Hz}$, sinusoidal) with synthesized $500 \mathrm{~Hz}, 2000 \mathrm{~Hz}$ and $5000 \mathrm{~Hz}$ sinusoidal waves. A deviation of 4,20 , and $50 \mathrm{~Hz}$ was used to add a jitter to the signal aiming at adapting the artificial signal to a "real" speech signal. In the following, "word length adapted chunks" of the resulting complex wave became an "envelope clone" of the spoken words used in this experiment: the real speech provided the amplitude "template", which was superimposed on the artificial signal. This procedure led to a completely artificial but complex amplitude and frequency modulated signal ("pseudo-speech"), which was comparable in complexity and length to the real speech signals with the aim to eliminate the pure sound analyzing part of language comprehension (Fig. 1).

\section{Activation Tasks and Experimental Procedure}

Participants were scanned with their eyes closed. They had to listen to five word blocks each containing 10 different concrete nouns, five blocks each containing 10 different abstract nouns and five blocks each containing 10 different pseudo-speech stimuli. Each of the activation blocks was randomly presented and anteceded and followed by a baseline resting condition. Moreover, another sixteen blocks each containing 10 distractor words were interspersed among critical stimulus blocks in order to avoid any mutual influence between them. Half of the participants heard the activation blocks in a different order. Based on a pilot study with six subjects the optimal time duration of an activation block was $30 \mathrm{~s}$. Stimulus onset asynchrony of the nouns was $3 \mathrm{~s}$. Scans were taken after each stimulus was presented in a 

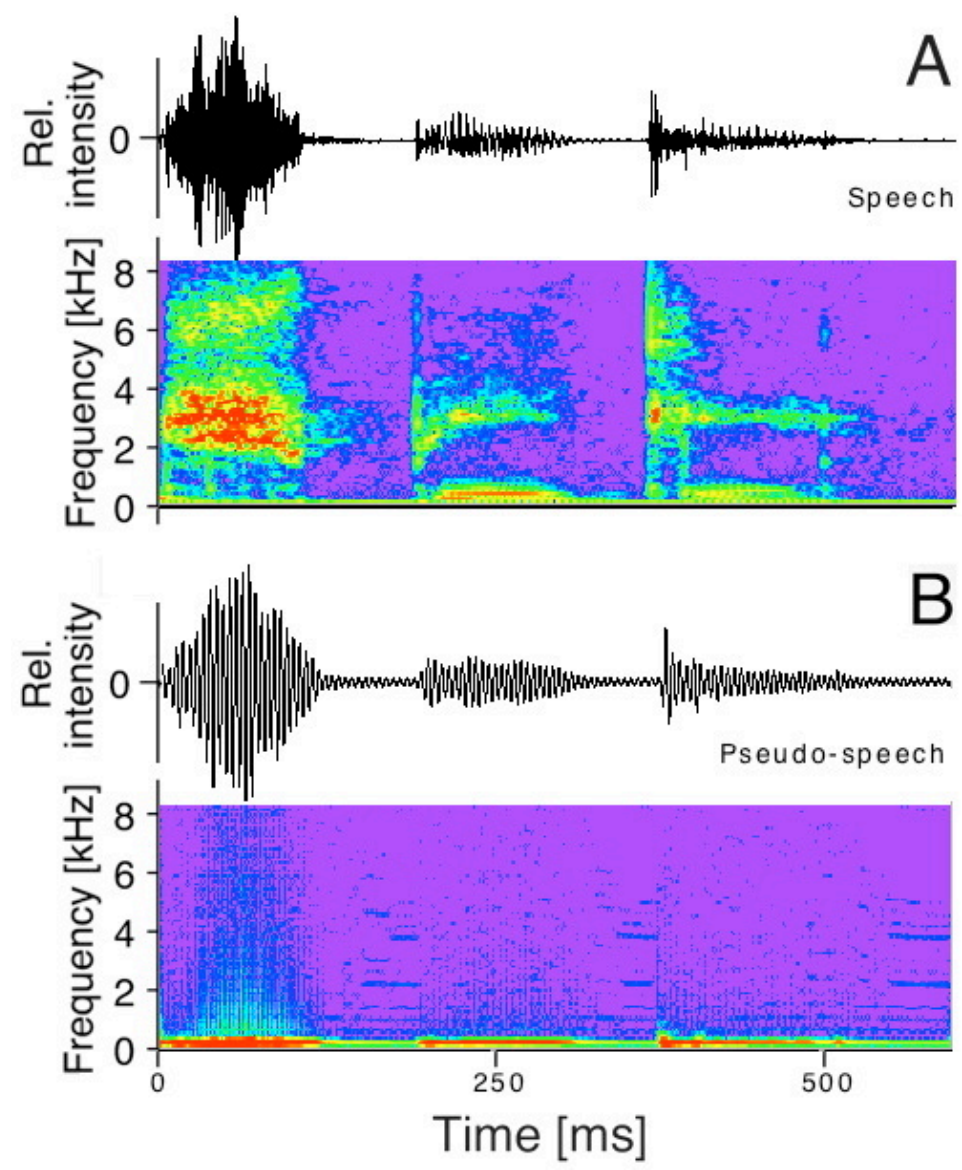

Fig. (1). Comparison of speech and artificially synthesized "pseudo-speech" in the intensity and frequency domain. A: An example of the spoken word "Spiegel" (mirror) as time-waveform (upper), and the spectrogramm (lower). B: The corresponding "pseudo-speech" signal consists of the same intensity envelope (upper) and length but is made up of "wordless" intensity and frequency modulated sinusoidal waves (lower).

silent period lasting $1.4 \mathrm{~s}$. Participants were not disturbed by loud background scanner noise during stimulus comprehension. During the unspecific baseline condition, which also lasted $30 \mathrm{~s}$, participants were requested to rest and relax. The participants were asked not to move during the scan, their heads were fixated.

The only instruction participants had was to listen carefully to the items presented. After the whole scanning session participants were examined on their strategies or impressions associated with word processing. Four participants reported that concrete nouns always elicited visual images, seven participants reported that about $70 \%$ of the concrete nouns elicited images, and only 3 reported that no visual images were elicited at all. Two persons reported on occasional visual images also for the abstract nouns. We tested the attention of the participants with a post-hoc questionnaire, on average $90 \%$ of the stimuli were correctly remembered.

\section{Data Acquisition}

MRI scans were carried out using a $1.5 \mathrm{~T}$ scanner (Siemens Magnetom Symphony, Erlangen, Germany) equipped with a standard head coil and with echoplanar imaging capability. To position the axial $\mathrm{T} 2 *$-weighted images along the AC-PC line, scout and sagittal T1-weighted images were obtained from each participant. To provide an anatomical reference and to exclude gross brain pathology, a T1weighted 3D-sequence (MPRAGE, TR $=11.1 \mathrm{~ms}$, TE $=4.3$ $\mathrm{ms}$, slice thickness $1.5 \mathrm{~mm}, \mathrm{FOV}=201 \times 230 \mathrm{~mm}$, matrix $224 \times 256$ ) was obtained from each participant.

Functional imaging volumes were acquired according to a block paradigm with each block lasting $30 \mathrm{~s}$. During each session there were 5 activation blocks per condition (concrete, abstract, pseudo-speech) so that the total duration of a session was 15 minutes. Volume scans were carried out at intervals of $3 \mathrm{~s}$ using a standard EPI sequence $(\mathrm{TR}=1600$ $\mathrm{ms}, \mathrm{TE}=50 \mathrm{~ms}$, flip angle $90^{\circ}, \mathrm{FOV}=192 \mathrm{~mm}$, matrix $64 \times 64)$. Each volume scan comprised 16 axial T2*-weighted MR-slices with a slice thickness of $7 \mathrm{~mm}$, covering the whole brain.

\section{Image Analysis}

The first two images of each session were discarded, to allow for $\mathrm{T} 1$ saturation effects during the first scans. The remaining images were processed using SPM. To correct for head movements, the images were first realigned using the SPM default algorithm. Prior to smoothing and group comparisons, anatomical differences were compensated for by spatial normalization and reslicing using the default settings and the standard stereotactic space, i.e. the MNI 
(Montreal Neurological Institute) brain. Then spatial smoothing was performed with a Gaussian kernel of $10 \mathrm{~mm}$ full-width at half-maximum (FWHM) to increase signal and anatomical conformity. A fixed-effects statistical analysis was performed on a voxel-by-voxel basis using the General Linear Model (GLM). Maps of $t$-statistics were corrected (FDR) for multiple comparisons at $p \leq 0.05$, and the minimum size of clusters displayed was 10 voxels. The MNI coordinates of the major activation clusters were transformed into the Talairach and Tournoux space [37] using a correction procedure [38] and then fed into the Talairach Daemon [39] to obtain anatomical projections of maximum activation. We report all brain activations above the chosen thresholds and report descriptively on those regions showing a trend ( $\mathrm{p} \leq 0.001$, uncorrected) where this contributes to the interpretation of our data.

Fixed-effects analyses assume that each participant makes the same, fixed contribution to the activation observed and therefore reduce random variations from participant to participant. Therefore results of these analyses are only interpretable for the 14 participants investigated. Therefore, in a second step a random-effects analysis (one sample $t$-test) was used. With regard to the small sample size, however, these analyses were performed on a voxelwise significance level of an uncorrected $p \leq 0.001$.

\section{RESULTS}

\section{Rating}

In order to obtain the participant's individual scores for the imageability and concrete-/abstractness of the nouns perceived they completed a self-rating scale after the MRI acquisition. On a 7-point rating scale participants rated concrete nouns with a mean score of $6.86 \pm 0.15$ for imagery and $6.67 \pm 0.43$ for concrete-/abstractness in contrast to the abstract nouns which they rated $1.69 \pm 1.01$ for imagery and $2.24 \pm 0.81$ for concrete-/abstractness. The difference in concrete-/abstractness and imagery between the concrete and abstract nouns was highly significant ( $t$-test, $2 p \leq 0.0001$ ). The higher standard deviation for the imagery and concrete/abstractness scores for abstract nouns indicated that this pool of participants rated concrete nouns in a more uniform way.

\section{Concrete Minus Abstract Nouns}

Fixed-effects analysis revealed more activation for the processing of concrete nouns in the left superior and medial frontal gyrus and the anterior cingulate (BA 10/12/32) in the 14 participants at a significance level of corrected $p \leq 0.05$ (Fig. 2). There was also a tendency towards a higher bilateral

Table 1. A: Fixed Effects Analysis. Brain Regions Showing Increased Activation for the Processing of Concrete vs. Abstract Nouns

\begin{tabular}{|c|c|c|c|c|c|c|c|}
\hline \multirow{2}{*}{ Hemis. } & \multirow{2}{*}{ Brain region } & \multirow{2}{*}{ Brodmann A. } & \multirow{2}{*}{$\mathbf{k}$} & \multirow{2}{*}{$\mathbf{Z}$} & \multicolumn{3}{|c|}{ Talairach Coordinates } \\
\hline & & & & & $\mathbf{x}$ & $\mathbf{y}$ & $\mathbf{z}$ \\
\hline - & Superior/ Middle Frontal Gyrus & $9 / 10 / 11 / 12 / 24 /$ & 1800 & 5.94 & -21 & 55 & 0 \\
\hline - & Medial Frontal Gyrus & $32 / 45 / 46$ & & 5.02 & -12 & 38 & -7 \\
\hline - & Anterior Cingulate & & & 4.93 & -9 & 26 & -6 \\
\hline Left & Precuneus & 19 & 34 & 4.21 & -42 & -74 & 34 \\
\hline Right & Postcentral Gyrus & $2 / 40$ & 271 & 4.04 & 59 & -21 & 45 \\
\hline Right & Inferior Parietal lobule & 40 & & 4.01 & 45 & -35 & 57 \\
\hline Left & Inferior Parietal lobule & 40 & 41 & 3.95 & -45 & -44 & 55 \\
\hline Left & Cerebellum, Culmen & & 80 & 3.91 & -36 & -51 & -25 \\
\hline Left & Gyrus fusiformis & $35(36 / 37)$ & & 3.39 & -27 & -27 & -21 \\
\hline Right & Middle Occipital Gyrus & 18 & 11 & 3.71 & 39 & -84 & 4 \\
\hline Left & Middle Occipital Gyrus & 18 & 38 & 3.49 & -36 & -90 & 2 \\
\hline Left & Postcentral & $1-3$ & 13 & 3.41 & -62 & -22 & 34 \\
\hline
\end{tabular}

Number of voxels $(\mathrm{k})$ and voxel $\mathrm{z}$ scores $(\mathrm{Z})$ as well as Talairach coordinates of the significant activation areas are illustrated (height threshold $\mathrm{T}=3.09, p \leq 0.001$, uncorrected for multiple comparisons; cluster size $>10)$.

B: Random Effects Analysis. Concrete vs. Abstract Nouns

\begin{tabular}{|c|c|c|c|c|c|c|c|}
\hline \multirow{2}{*}{ Hemisphere } & \multirow{2}{*}{ Brain Region } & \multirow{2}{*}{ Brodmann Area } & \multirow{2}{*}{$\mathbf{k}$} & \multirow{2}{*}{$\mathbf{Z}$} & \multicolumn{3}{|c|}{ Talairach Coordinates } \\
\hline & & & & & $\mathbf{x}$ & $\mathbf{y}$ & $\mathbf{z}$ \\
\hline \multirow[t]{2}{*}{ Left } & \multirow[t]{2}{*}{ Medial Frontal Gyrus } & \multirow[t]{2}{*}{$32 / 10$} & \multirow[t]{2}{*}{73} & 3.68 & -6 & 47 & 0 \\
\hline & & & & 3.43 & -12 & 44 & 14 \\
\hline Left & Inferior Frontal Gyrus & $45 / 46$ & 4 & 3.22 & -42 & 30 & 10 \\
\hline \multirow[t]{3}{*}{ Right } & \multirow[t]{3}{*}{ Middle Frontal Gyrus } & \multirow[t]{3}{*}{46} & 5 & 3.55 & 27 & 33 & 15 \\
\hline & & & 1 & 3.16 & 27 & 46 & -5 \\
\hline & & & 1 & 3.10 & 36 & 47 & 6 \\
\hline Right & Inferior Frontal Gyrus & $45 / 46$ & 1 & 3.22 & 33 & 26 & 1 \\
\hline
\end{tabular}

Height threshold $\mathrm{T}=3.85, p \leq 0.001$, uncorrected for multiple comparisons. 
activation with a left hemispheric preponderance in the superior and middle frontal gyri, the medial frontal gyri and the anterior cingulate, the left precuneus (BA 19), bilateral inferior parietal lobules (BA 40) and bilateral postcentral gyri (Table 1A). Further, increased activation was found in bilateral middle occipital gyri (BA 18), the left fusiform gyrus and the left cerebellum (Fig. 2).
An additional random effects analysis showed only a very slight difference in brain activation between the processing of concrete and abstract nouns. Processing concrete nouns induced more activation in left medial (BA 32/10) and inferior (BA 45/46) frontal gyrus, and in the right middle and inferior frontal gyrus (BA 45/46) (Table 1B, Fig. 3). No cortical brain region showed more activation for abstract nouns processing.

\section{Concrete minus Abstract Nouns}

L
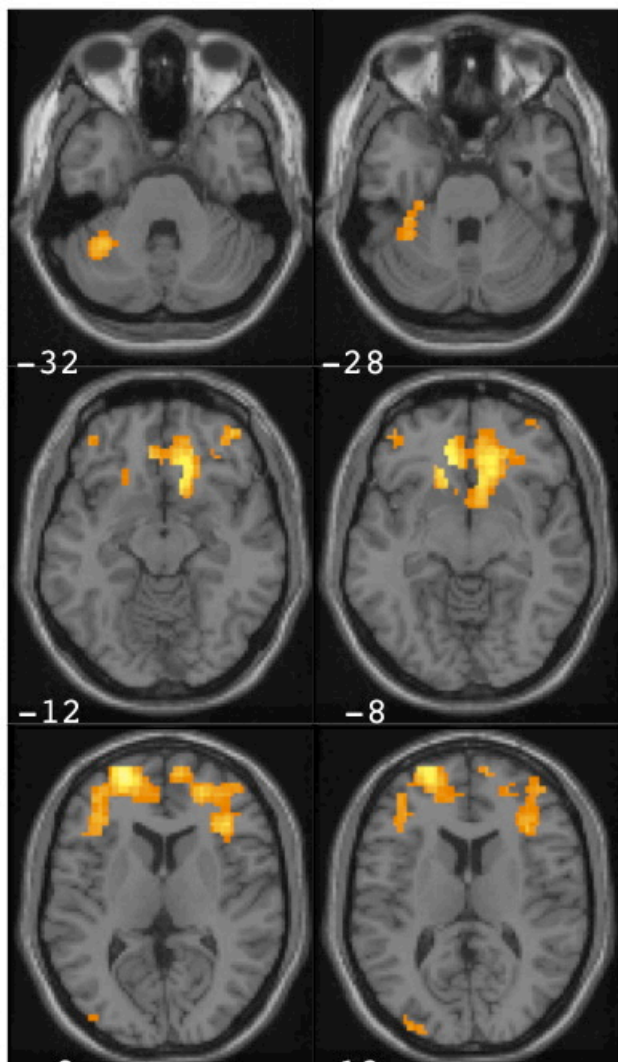

$+8$

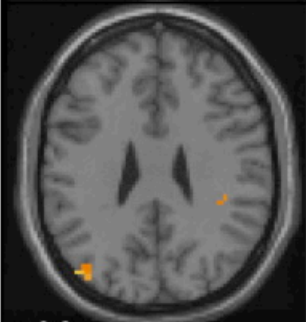

$+28$

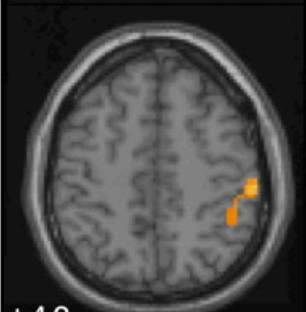

$+48$

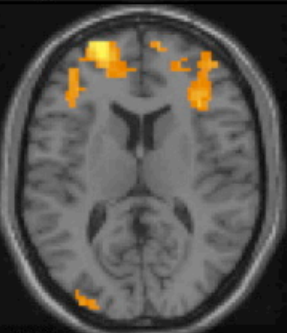

$+12$

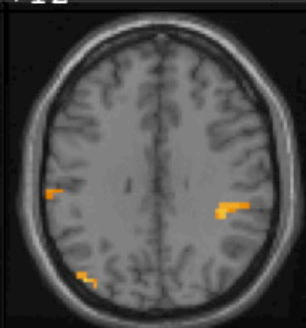

$+32$

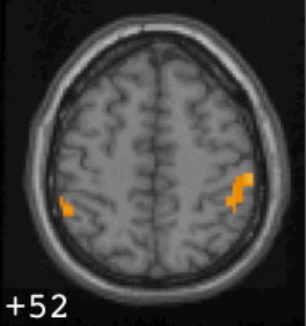

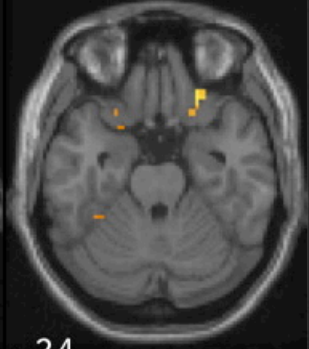

$-24$
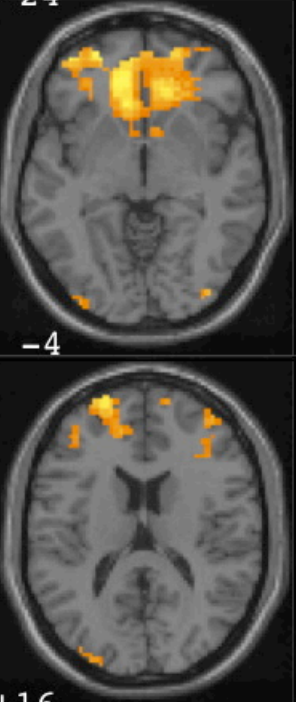

$+16$

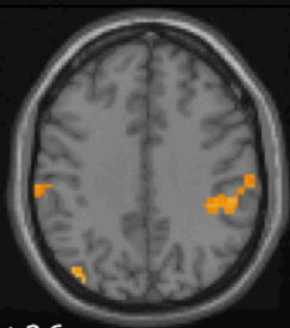

$+36$

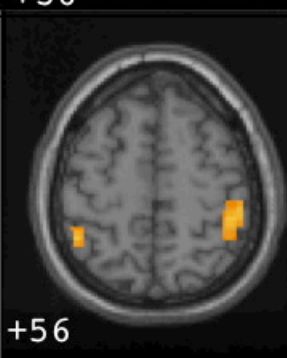

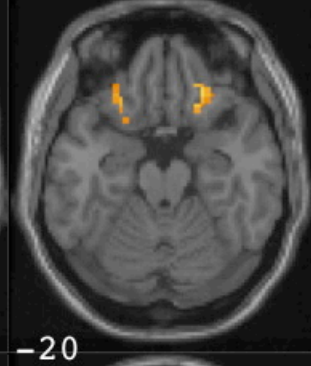

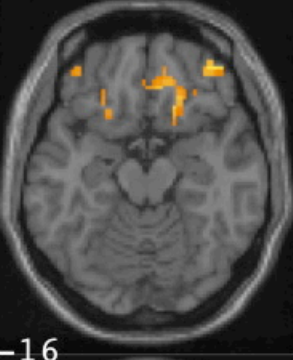

$-16$
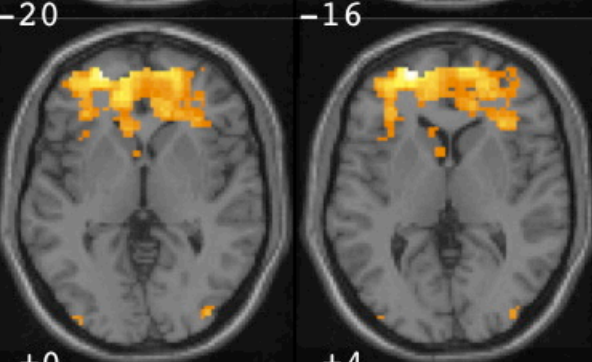

$+0$

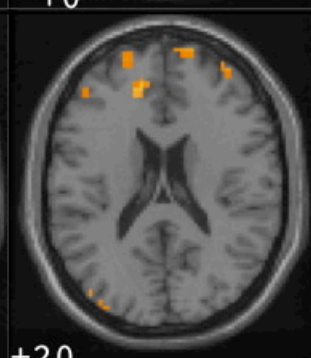

$+4$

$+20$

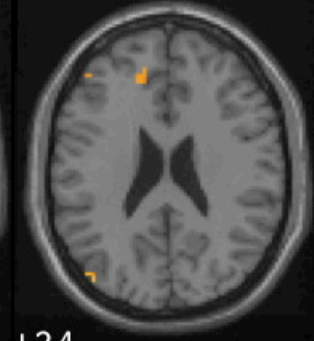

$+24$
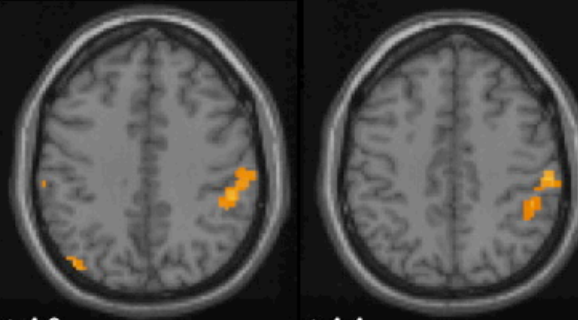

$+44$

$+40$

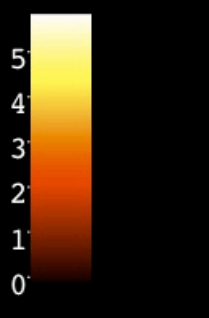

Fig. (2). fMRI activation maps for the processing of concrete compared to abstract nouns revealed with fixed effects analyses. Planes are sequential axial slices in the z-direction from - 32 to $+60 \mathrm{~mm}$ (slice thickness: $4 \mathrm{~mm}, \mathrm{~T}=0.0-5.0$ ). L denotes the left hemisphere; $\mathrm{R}$ denotes the right hemisphere. Concrete nouns show tendentially higher activation than abstract nouns in distributed frontal, parietal and occipital brain regions. 
Concrete Nouns minus

\section{Abstract Nouns}

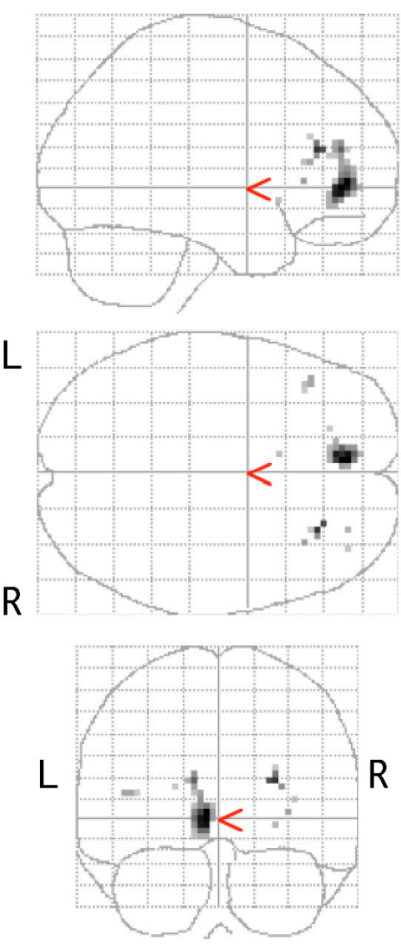

Fig. (3). SPM glass brain projections of regions showing more activation for the comprehension of concrete minus abstract nouns (voxel $\mathrm{z}$ score $>3.85, p \leq 0.001$, uncorr.,) revealed with random effects analyses. $\mathrm{L}$ denotes the left hemisphere; $\mathrm{R}$ denotes the right hemisphere.

\section{Abstract Minus Concrete Nouns}

Fixed-effects analysis revealed no increased activity for the processing of abstract nouns at a significance level of corrected $p \leq 0.05$.

\section{Concrete Nouns versus Pseudo-Speech}

Comprehension of concrete nouns relative to the perception of pseudo-speech stimuli elicited more activation in various distributed cortical brain regions of the frontal, temporal, parietal, and limbic lobes. Fixed-effect analysis revealed more activation in the left medial frontal gyrus (BA 6), bilateral cingulate gyri (BA 24/32), bilateral insulae, bilateral middle and inferior frontal gyri at a significance level of corrected $p \leq 0.05$. There was also a tendency towards more activation in the left precuneus (BA 7), bilateral middle temporal gyri (BA 21), bilateral inferior parietal lobules including the right supramarginal gyrus (BA 40), right superior parietal lobule (BA 7), the right anterior cingulate and the left cerebellum (Table 2; Fig. 4, upper panel; Fig. 5, left panel).

\section{Abstract Nouns versus Pseudo-Speech}

In contrast to concrete nouns, the comprehension of abstract nouns compared to the perception of pseudo-speech stimuli elicited increased activation within fewer brain regions mainly of the temporal and frontal lobes. Fixedeffect analysis revealed more activation in the left middle and inferior temporal gyrus (BA 20/21), bilateral medial frontal gyri and the left middle frontal gyrus (BA 9) at a significance level of corrected $p \leq 0.05$. There was also a tendency towards more activation in the right cingulate gyrus (BA 32), right middle frontal gyrus (BA 9), the right middle temporal gyrus (BA 20/21) and the right lingual gyrus/

Table 2. Fixed Effects Analysis: Concrete Nouns vs. Pseudo-Speech

\begin{tabular}{|c|c|c|c|c|c|c|c|}
\hline \multirow{2}{*}{ Hemis. } & \multirow{2}{*}{ Brain region } & \multirow{2}{*}{ Brodmann A. } & \multirow{2}{*}{$\mathbf{k}$} & \multirow{2}{*}{$\mathbf{Z}$} & \multicolumn{3}{|c|}{ Talairach Coordinates } \\
\hline & & & & & $\mathbf{x}$ & $\mathbf{y}$ & $\mathbf{z}$ \\
\hline Left & Medial Frontal Gyrus & 6 & 732 & 6.57 & -3 & 17 & 46 \\
\hline Right & Cingulate Gyrus & $24 / 32$ & & 5.80 & 9 & 28 & 29 \\
\hline Left & Cingulate Gyrus & $24 / 32$ & & 5.08 & -6 & 30 & 26 \\
\hline Right & Insula, Inferior Frontal Gyrus & $46 / 47$ & 545 & 5.69 & 33 & 23 & 2 \\
\hline Right & Middle Frontal Gyrus & $9 / 10$ & & 5.18 & 42 & 51 & 20 \\
\hline Left & Middle/Inferior Frontal Gyrus & $8 / 9 / 10 / 46 / 47$ & 498 & 5.04 & -50 & 28 & 29 \\
\hline Left & Insula & & & 4.85 & -36 & 17 & -1 \\
\hline Left & Precuneus & 7 & 99 & 4.19 & -30 & -68 & 42 \\
\hline Right & Middle Temporal Gyrus & 21 & 16 & 4.13 & 59 & -1 & -13 \\
\hline Left & Middle Temporal Gyrus & 21 & 37 & 4.12 & -59 & -32 & -6 \\
\hline Left & Inferior Parietal Lobule & 40 & 65 & 4.03 & -56 & -48 & 38 \\
\hline Left & Cerebellum, Anterior lobe & & 23 & 3.95 & -33 & -57 & -27 \\
\hline Right & Inferior Parietal Lobule & 40 & 57 & 3.65 & 53 & -36 & 38 \\
\hline Right & Supramarginal Gyrus & 40 & & 3.54 & 62 & -42 & 33 \\
\hline Right & Superior Parietal Lobule & 7 & 18 & 3.50 & 33 & -65 & 47 \\
\hline Right & Anterior Cingulate & 32 & 12 & 3.39 & 12 & 41 & -2 \\
\hline
\end{tabular}

Height threshold $\mathrm{T}=3.09, p \leq 0.001$, uncorrected for multiple comparisons; cluster size $>10$. 


\section{$\mathrm{L}$ Concrete Nouns minus Pseudo-speech}
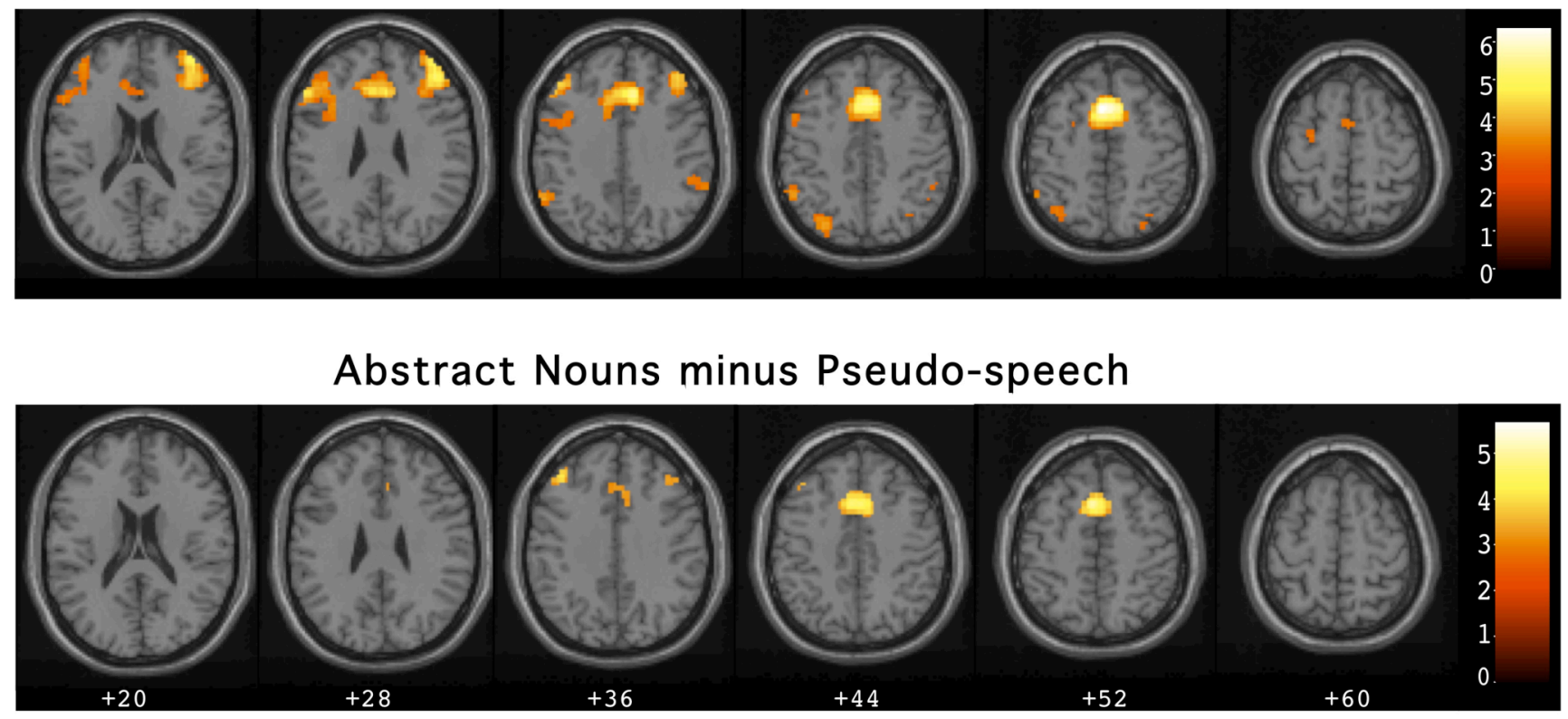

Fig. (4). fMRI activation maps: average brain activation for the processing of concrete (upper panel) and abstract nouns (lower panel), both compared to the processing of pseudo-speech stimuli revealed with fixed effects analyses. Data are presented as planes of axial slices in the z-direction ( +20 to $+60 \mathrm{~mm}$, thickness $4 \mathrm{~mm}, \mathrm{~T}=0.0-6.0)$. Only every second slice is shown. $\mathrm{L}$ denotes the left, $\mathrm{R}$ the right hemisphere. Compared to the perception of pseudo-speech stimuli comprehension of concrete nouns shows tendentially increased activation in more and distributed brain regions than that of abstract nouns.

fusiform gyrus (BA 37) (Table 3; Fig. 4, lower panel; Fig. 5, right panel).

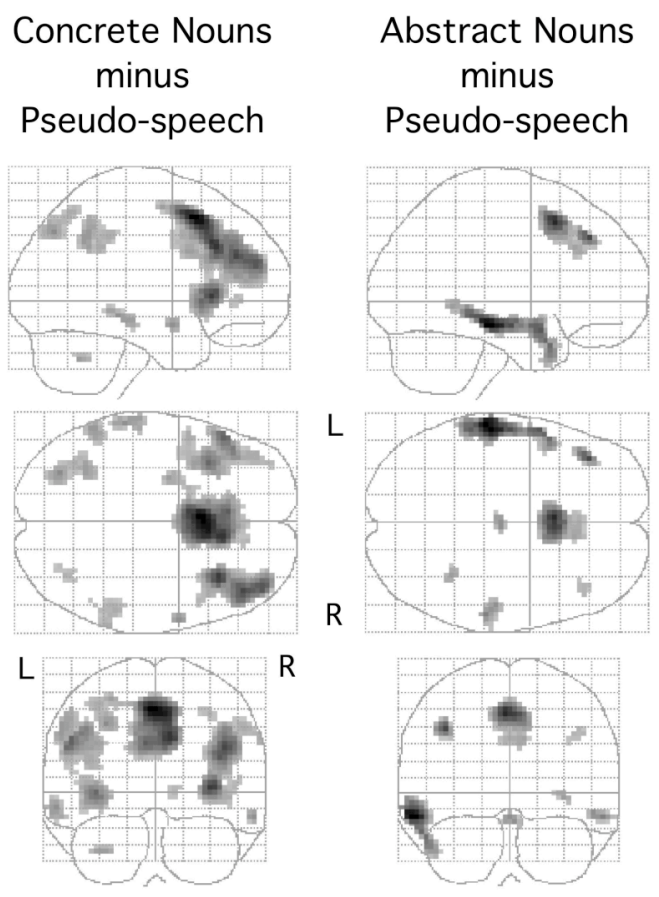

Fig. (5). SPM glass brain projections of regions showing more activation for the comprehension of concrete (left panel) and abstract nouns (right panel) relative to the perception of pseudospeech stimuli (voxel z score $>3.09, p \leq 0.001$, uncorr.; cluster size $>10$ voxels) revealed with random effects analyses. L denotes the left, $\mathrm{R}$ the right hemisphere.

\section{DISCUSSION}

\section{Concrete versus Abstract Nouns}

The main result of the random effects analysis was that processing of concrete nouns was associated with increased ventromedial prefrontal activation compared to the processing of abstract nouns. The fixed effects analysis revealed that in these 14 participants the most prominent differences between the comprehension of concrete and abstract nouns were found in left frontopolar regions (BA 9-12) and the medial frontal gyrus (BA 32/10) of prefrontal cortex showing more activation for concrete nouns. Additionally, more activation in the left inferior frontal gyrus (BA 45/46), anterior portion of the insula and the right middle frontal gyrus (BA 46) has been elicited by processing concrete nouns. The anterior cingulate cortex was (BA 24/32) activated by both word types, whereas concrete nouns were associated with more activation.

A participation of different regions of the ventromedial prefrontal cortex in the processing of concrete and abstract nouns has been reported in studies using PET and fMRI although results were divergent and the application of different cognitive tasks makes comparisons difficult [40]. In our study comprehension of concrete compared to abstract nouns elicited more activation of the ventromedial prefrontal cortex, in particular in the left hemisphere. This finding stays in contrast to previous PET and fMRI studies on concrete and abstract nouns processing. For instance, lexical decision tasks for visually presented concrete and abstract nouns elicited higher activation of the left inferior frontal gyrus [16] and bilateral inferior frontal gyrus for abstract nouns [26]. In correspondence with these findings, Abdullaev et al. 
Table 3. Fixed Effects Analysis: Abstract Nouns vs. Pseudo-Speech

\begin{tabular}{|c|c|c|c|c|c|c|c|}
\hline \multirow{2}{*}{ Hemis. } & \multirow{2}{*}{ Brain Region } & \multirow{2}{*}{ Brodmann A. } & \multirow{2}{*}{$\mathbf{k}$} & \multirow{2}{*}{$\mathbf{Z}$} & \multicolumn{3}{|c|}{ Talairach Coordinates } \\
\hline & & & & & $\mathbf{x}$ & $\mathbf{y}$ & $\mathbf{z}$ \\
\hline Left & Middle/Inferior Temporal Gyrus & $20 / 21$ & 242 & 5.67 & -56 & -24 & -11 \\
\hline Left & Medial Frontal Gyrus & 6 & 259 & 5.21 & -3 & 17 & 43 \\
\hline Right & Medial Frontal Gyrus & 6 & & & & & \\
\hline Right & Cingulate Gyrus & 32 & & 3.61 & 9 & 28 & 29 \\
\hline Right & Middle Temporal Gyrus & $20 / 21$ & 34 & 4.06 & 56 & -27 & -11 \\
\hline Right & Lingual Gyrus/Fusiform Gyrus & 37 & 13 & 3.72 & 33 & -47 & 0 \\
\hline Right & Middle Frontal Gyrus & 9 & 15 & 3.33 & 42 & 34 & 34 \\
\hline
\end{tabular}

Height threshold $\mathrm{T}=3.09, p \leq 0.001$, uncorrected for multiple comparisons; cluster size $>10$.

[41] reported on neurons in the left prefrontal cortex (Brodmann areas 10 and 46) responding only during the presentation of abstract and not concrete nouns in a patient with implanted intracortical electrodes who performed a lexical decision task. However, higher ventromedial prefrontal activation for abstract nouns during lexical decision may demonstrate that it is more demanding to decide whether an abstract word is a member of a certain category than to do this for concrete ones. Thus, in this case prefrontal activation is probably associated with task difficulty rather than inherent conceptual differences between concrete and abstract nouns. The higher activation of left prefrontal regions by concrete nouns in our study may reflect a difference in the integration of multimodal concepts underlying these word types rather than their manipulation in order to perform a certain task. In particular, it seems possible that passive listening without an explicit task allows participant analyzing the affective significance of concepts [40], which may be easier and less variable for concrete nouns. Probably, a passive listening task is not suitable to canalize the higher variability in processing abstract items and results in less coherent brain activation.

Generally, activation of the left prefrontal cortex seems to be associated with semantic processing of stimuli across many different cognitive tasks independently of stimulus modality (visual, auditorily, pictoral). Moreover, activation in the so-called left frontal semantic area occurs during both intentional and incidental paradigms. It seems that the left prefrontal cortex is activated by the extend of semantic knowledge that is held in working memory in order to process meaningful stimuli [40]. Semantic encoding differs for concrete and abstract nouns since concrete material can be categorized and organized more easily because of multiple sources (e.g., imagery) that support the process. In correspondence with our present findings an fMRI study dealing with memory encoding of visually presented concrete and abstract words, demonstrated more activation in left prefrontal regions for concrete words which was interpreted in terms of increased contextual support for them [18]. The semantic classification whether a word was concrete or abstract activated the left inferior frontal gyrus (BA 45) [17] and bilateral prefrontal cortex (BA 10/46) both for visually and auditorily presented nouns [42].
Our results fit well to previous findings showing higher EEG coherence between left frontal electrodes for concrete compared to abstract nouns for both the auditory and the visual modality which probably can be correlated with more elaborate encoding strategies for concrete compared to abstract nouns [43, 44]. EEG studies examining the effect of concreteness on event-related potentials (ERPs) reported on a greater effect on the ERPs to concrete than abstract words which was more frontally distributed $[11,45,46]$. This effect was larger in a semantic categorization task than in a lexical decision task indicating that semantic properties of the words influence the extend of the differences. Thus, it has been hypothesized, that the activation of the left inferior prefrontal cortex may reflect a "mental imagery subsystem", responsible for holding mental images in semantic working memory [46].

Numerous other studies demonstrated the participation of different regions of the prefrontal cortex in several different cognitive tasks. Comparing the data it is almost impossible to specify the function of a frontal region given the fact that one and the same bilateral prefrontal network is recruited by several different cognitive demands [47]. It is well known that massive neuroanatomical projections of the medial dorsal nucleus of the thalamus to frontal areas (BA 8/9/10/$11 / 12$ ) exist. On the other hand, this nucleus receives projections from other thalamic nuclei, amygdala and multisensory association cortex [48]. Thus, it is most likely, that in these frontal regions the integration of different cognitive and emotive information streams takes place such as the relation of the noun's meaning to its semantic context, the integration of visual, auditory, tactile and probably other sensory images and the activation of sensomotoric and manipulation-related context in order to form the semantic notion of a concrete noun. This is in accordance with findings indicating that left frontal regions may coordinate the sensory and semantic processes occurring in posterior temporal and parietal areas [40]. In particular, the frontopolar prefrontal cortex (BA 10) is engaged in monitoring and evaluating internally generated information and post-retrieval products, whereas the dorsolateral prefrontal cortex (BA 9/46) is specifically involved in monitoring processes during episodic retrieval [49].

In the present investigation no further significant differences for the direct comparison of concrete and abstract 
nouns were found besides the activation of the prefrontal cortex. Nevertheless, recent studies demonstrated more activation in left and right temporal cortex for abstract nouns $[22,23,26,27]$. This activation has been interpreted in terms of abstract nouns demanding sentential context for the comprehension of their meaning. However, increased activation of the right temporal cortex was associated with increasing task difficulty and attention to the task [50]. This goes in line with the findings of Friederici et al. [17] who showed that activation in the left temporal lobe varied as a main effect of task (semantic versus syntactic) whereas activation in the left inferior frontal region was modulated by both factors, task and word concreteness. In addition, processing concrete nouns elicited more activation in the left inferior temporal/ fusiform gyrus [16, 22-24, 27] and occipital association cortex $[23,24,29]$. Activation of these temporo-occipital regions was specifically associated with the active manipulation of concrete nouns (for instance intentional visual imagery) which stays in contrast to the more passive paradigm of our present study. To our knowledge, none of these studies investigated differences in passive but attentive listening to concrete and abstract nouns without any explicit task instruction. Therefore, findings in previous studies may often reflect task-related manipulation of concrete or abstract nouns, necessary to perform certain cognitive operations.

\section{Concrete and Abstract Nouns versus Pseudo-Speech}

The comparison between listening to either concrete or abstract nouns and pseudo-speech aimed at supporting the findings of the direct comparison between both word types. On the one hand, comparing the real speech with the pseudospeech signals may eliminate primary auditory processes and non-specific executive functions mediating attention and arousal not important for this study. On the other hand, it may demonstrate language-relevant activation common to both word types such as matching of acoustic properties to phonetic features and the activation and selection of appropriate lexical entries in semantic long-term memory on the basis of sounds.

Compared with pseudo-speech concrete and abstract nouns activated the same specific brain regions as in the direct comparison, as has already been discussed in the previous sections. However, the comparison with pseudospeech also yielded important additional findings. First, most of the previous hemodynamic studies on auditory word comprehension showed activation of bilateral superior temporal cortices [e.g., 51]. Comparing the comprehension of nouns with pseudo-speech elicited no such activation in our study. Therefore, the creation of a baseline able to extinguish the prelexical analysis of speech was successful. Second, there were brain regions activated by both word types essentially having identical peak coordinates. These regions were the medial frontal gyri (BA 6) with a more extended voxel cluster for concrete nouns, the anterior cingulate gyrus (BA 32) and bilateral middle temporal gyri (BA 21) with abstract nouns yielding activation extending into the inferior temporal gyrus (BA 20). However, this broader activation, particularly of the left temporal cortex, was not found in the direct comparison.

Concrete as well as abstract nouns activated the left and right medial frontal gyrus (BA 6), which is involved in several different tasks and seems to play a multifunctional role in language [40]. Its anterior portion also activated in our study has been related to word comprehension and semantic processing [40], picture and auditory word recall [52], and verbal working memory [53]. Similarly, activation of the anterior cingulate gyrus has been found in a variety of different cognitive tasks and probably reflects the attention to auditory linguistic stimuli and affective concept analysis in this study [40, 54]. Both concrete and abstract nouns activated bilateral middle temporal gyri, whereby abstract nouns activated a broader cluster of voxels extending into the left inferior temporal gyrus. The middle temporal gyrus is associated with semantic retrieval for both verbal and nonverbal material [55] and is more involved in the semantic processing of auditory than visual words [42] linking modality specific word forms with the distributed associations that give them meaning. The activation tends to be bilateral in auditory tasks and left lateralized in visual tasks. Activation in this region was more specifically associated with the semantic nature of a concrete/abstract classification than the word concreteness per se [17]. In a fMRI study Whatmough et al. [23] found activation common to concrete and abstract nouns in the left middle and inferior temporal gyri. They suggested a role for general semantic knowledge mediated by this region which has to be distinguished from the attributes of objects such as their physical structure.

In sum, in order to form the semantic notion of a concrete noun the relation of the noun's meaning to its semantic context, the integration of visual, auditory, tactile and probably other sensory images and the activation of sensomotoric and manipulation-related context has to be performed. It is most likely, that the integration of different cognitive and emotive information streams takes place in ventromedial prefrontal regions. Higher prefrontal activation for concrete compared to abstract nouns may reflect the integration of a higher amount of different modalities and sensory experiences. This is supported by the existence of massive neuroanatomical projections of the medial dorsal nucleus of the thalamus to frontal areas (BA 8/9/10/11/12). In addition, this nucleus receives projections from other thalamic nuclei, amygdala and multisensory association cortex [48], which is in accordance with findings indicating that left frontal regions may coordinate the sensory and semantic processes occurring in posterior temporal and parietal areas [40]. Probably, all participants of this study have to perform this integration or filtering process but their associative relation to each concept is individual and is cancelled by the averaging process. The usage of a pseudo-speech baseline allowed us to demonstrate common neuronal networks involved in the comprehension of both word types without the activation of primary auditory processes and non-specific executive functions mediating attention and arousal.

\section{ACKNOWLEDGEMENT}

This project was supported by the German Science Foundation (Excellence Cluster 277, SFB-673). For her help with data acquisition we wish to thank Mrs. I. Ollek. We would like to thank Jan de Ruiter for helpful comments on an earlier version of the manuscript. 


\section{REFERENCES}

[1] Ewald P. Konkreta versus Abstrakta. Zur semantischen Subklassifikation deutscher Substantive. Sprachwissenschaft 1992; 17: 259-81.

[2] Bloom P. How children learn the meanings of words. Cambridge: MIT Press 2000.

[3] Paivio A. Dual coding theory: Retrospect and current status. Can J Psychol 1991; 45: 255-87.

[4] Schwanenfluegel PJ, Shoben EJ. Differential context effects in the comprehension of abstract and concrete verbal materials. J Exp Psychol Learn Mem Cogn 1983; 9: 82-102.

[5] Walker I, Hulme Ch. Concrete words are easier to recall than abstract words: evidence for a semantic contribution to short-term serial recall. J Exp Psychol Learn Mem Cogn 1999; 25: 1256-71.

[6] Crutch SJ, Warrington EK. Abstract words are represented in an associative network whilst concrete words are represented in a categorical network. Brain Lang 2004; 91: 13-4.

[7] Katz RB, Goodglass H. Deep dysphasia: analysis of a rare form of repetition disorder. Brain Lang 1990; 39: 153-85.

[8] Tyler LK, Moss HE, Jennings F. Abstract word deficits in Aphasia: evidence from semantic priming. Neuropsychol 1995; 9: 354-63.

[9] Crutch SJ, Connell S, Warrington EK. The different representational frameworks underpinning abstract and concrete knowledge: evidence from odd-one-out judgements. Q J Exp Psychol (Colchester) 2009; 62: 1377-90.

[10] Weiss S, Rappelsberger P. EEG coherence within the $13-18 \mathrm{~Hz}$ band as a correlate of a distinct lexical organisation of concrete and abstract nouns in humans. Neurosci Lett 1996; 209: 17-20.

[11] Swaab TY, Baynes K, Knight RT. Separable effects of priming and imageability on word processing: an ERP study. Cogn Brain Res 2002; 15: 99-103.

[12] Binder JR, Westbury CF, McKiernan KA, Possing ET, Medler DA. Distinct brain systems for processing concrete and abstract concepts. J Cogn Neurosci 2005; 17: 905-17.

[13] Papagno C, Fogliata A, Catricala E, Miniussi C. The lexical processing of abstract and concrete nouns. Brain Res 2009; 1263: 78-86.

[14] Mecklinger A, Friederici AD. Elektrophysiologische dissoziationen beim Einpraegen abstrakter und konkreter woerter. Z Exp Psychol 1997; 1: 62-81.

[15] Holcomb PJ, Kounios J, Anderson JE, West WC. Dual-coding, context-availability, and concreteness effects in sentence comprehension: an electrophysiological investigation. J Exp Psychol Learn Mem Cogn 1999; 25: 721-42.

[16] Fiebach CJ, Friederici AD. Processing concrete words: fMRI evidence against a specific right-hemisphere involvement. Neuropsychol 2003; 42: 62-70.

[17] Friederici A, Opitz B, von Cramon DY. Segregating semantic and syntactic aspects of processing in the human brain: an fMRI investigation of different word types. Cerebr Cortex 2000; 10: 698705.

[18] Jessen F, Heun R, Erb M, et al. The concreteness effect: Evidence for dual coding and context availability. Brain Lang 2000; 74: 10312 .

[19] Noppeney U, Price CJ. Retrieval of abstract semantics. NeuroImage 2004; 22: 164-70.

[20] Ghio M, Tettamanti M. Semantic domain-specific functional integration for action-related vs. abstract concepts. Brain Lang 2010; 112: 223-32.

[21] Hoffman P, Jefferies E, Lambon RMA. Ventrolateral prefrontal cortex plays an executive regulation role in comprehension of abstract words: convergent neuropsychological and repetitive TMS evidence. J Neurosci 2010; 30: 15450-6.

[22] Mellet E, Tzourio N, Denis M, Mazoyer B. Cortical anatomy of mental imagery of concrete nouns based on their dictionary definition. NeuroReport 1998; 9: 803-8.

[23] Whatmough C, Verret L, Fung D, Chertkow H. Common and contrasting areas of activation for abstract and concrete concepts: An $\mathrm{H}_{2}{ }^{15} \mathrm{O}$ PET study. J Cog Neurosci 2004; 16: 1211-26.

[24] D'Esposito M, Detre JA, Aguirre GK, et al. A functional MRI study of mental image generation. Neuropsychol 1997; 35: 725-30.

[25] Sabsevitz DS, Medler DA, Seidenberg M, Binder JR. Modulation of the semantic system by word imageability. Neuroimage 2005; 27: $188-200$
[26] Kiehl KA, Liddle PF, Smith AM, Mendreck A, Forster BB, Hare $\mathrm{RD}$. Neural pathways involved in the processing of concrete and abstract words. Hum Brain Map 1999; 7: 225-33.

[27] Wise RJS, Howard D, Mummery CJ, et al. Noun imageability and the temporal lobes. Neuropsychol 2000; 38: 985-94.

[28] Mestres-Misse A, Munte TF, Rodriguez-Fornells A. Functional neuroanatomy of contextual acquisition of concrete and abstract words. J Cogn Neurosci 2009; 21: 2154-71.

[29] Kansaku K, Schimoyama I, Nakajima Y, et al. Functional magnetic resonance imaging during recognition of written words: chinese characters for concrete objects versus abstract concepts. Neurosci Res 1998; 30: 361-4.

[30] Klostermann EC, Kane AJ, Shimamura AP. Parietal activation during retrieval of abstract and concrete auditory information. Neuroimage 2008; 40: 896-901.

[31] Scott SK, Wise RJS. Functional imaging and language: a critical guide to methodology and analysis. Speech Commun 2003; 41: 721.

[32] Mueller HM, Weiss S, Rappelsberger P. EEG coherence analysis of auditory sentence processing. In: Witte $\mathrm{H}, \mathrm{Zwiener} \mathrm{U}$, Schack B, Doering, A, Eds. Quantitative and topological EEG and MEG analysis. Jena: Universitaetsverlag 1997, pp. 429-31.

[33] Woermann FG, Jokeit H, Luerding R, et al. Language lateralization by Wada test and fMRI in 100 patients with epilepsy. Neurology 2003; 61: 699-701.

[34] Baayen RH, Piepenbrock R, Gulikers L. The CELEX Lexical Database. Linguistic Data Consortium Philadelphia: University of Pennsylvania, 1995.

[35] Baschek I-L, Bredenkamp J, Oehrle B, Wippich W. Bestimmung der Bildhaftigkeit (I), Konkretheit, (C) und der Bedeutungshaltigkeit (m') von 800 Substantiven. Z Exp Psychol 1977; 24: 353-96.

[36] Offe H, Anneken G, Kessler E. Normen fuer die konkretheits- und vorstellbarkeitseinschaetzungen von 234 substantiven. Psychologische Beitraege 1981; 23: 65-85.

[37] Talairach J, Tournoux P. Co-planar stereotaxic atlas of the human brain. New York: Thieme 1988.

[38] Brett M, Lancaster J, Christoff K. Using the MNI brain with the Talairach atlas. NeuroImage 2001; 13: S85.

[39] Lancaster JL, Woldorff MG, Parsons LM, et al. Automated Talairach atlas labels for functional brain mapping. Hum Brain Map 2000; 10: 120-31

[40] Binder JR, Desai RH, Graves WW, Conant LL. Where is the semantic system? A critical review and meta-analysis of 120 functional neuroimaging studies. Cereb Cortex 2009; 19: 2767-96.

[41] Abdullaev YG, Bechtereva NP. Neuronal correlate of the higherorder semantic code in human prefrontal cortex in language tasks. Int J Psychophysiol 1993; 14: 167-77.

[42] Chee MWL, O'Craven KM, Bergida R, Rosen BR, Savoy R. Auditory and visual word processing studied with fMRI. Hum Brain Map 1999; 7: 15-28

[43] Weiss S, Rappelsberger P. Left frontal EEG coherence reflects modality independent language processes. Brain Topogr 1998; 11: $33-42$.

[44] Weiss S, Mueller HM. The contribution of EEG coherence to the investigation of language. Brain Lang 2003; 85: 325-34

[45] Kounios J, Holcomb PJ. Concreteness effects in semantic processing: ERP evidence supporting dual-coding theory. J Exp Psychol Learn Mem Cogn 1994; 20: 804-23.

[46] West WC, Holcomb PJ. Imaginal, semantic, and surface-sevel processing of concrete and abstract words: An electrophysiological investigation. J Cog Neurosci 2000; 12: 1024-37.

[47] Duncan J, Owen AM. Common regions of the human frontal lobe recruited by diverse cognitive demands. TINS 2000; 23: 475-83.

[48] Creutzfeldt OD. Cortex Cerebri. Berlin: Springer 1983.

[49] Christoff K, Gabrieli JDE. The frontopolar cortex and human cognition: Evidence for a rostrocaudal hierarchical organization within the human prefrontal cortex. Psychobiology 2000; 28: 16886.

[50] Price CJ, Wise RJ, Watson JD, Patterson K, Howard D, Frackowiak RS. Brain activity during reading. The effects of exposure duration and task. Brain 1994; 117: 1255-69.

[51] Price CJ. The anatomy of language: contributions from functional neuroimaging. J Anat 2000; 197: 335-59. 
[52] Buckner RL, Corbetta M, Schatz J, Raichle ME, Petersen SE. Preserved speech abilities and compensation following prefrontal damage. Proc Natl Acad Sci USA 1996; 93: 1249-53.

[53] Fiez JA, Raife EA, Balota DA, Schwarz JP, Raichle ME, Petersen SE. A positron emission tomography study of the short-term maintenance of verbal information. J Neurosci 1996; 16: 808-22.
[54] Benedict RH, Shucard DW, Santa Maria MP, et al. Covert auditory attention generates activation in the rostral/dorsal anterior cingulate cortex. J Cogn Neurosci 2002; 14: 637-45.

[55] Cabeza R, Nyberg L. Imaging cognition II: an empirical review of 275 PET and fMRI studies. J Cog Neurosci 2000; 12: 1-47.

Received: September 02, 2011

Revised: October 25,2011

Accepted: October 28, 2011

(C) Weiss et al.; Licensee Bentham Open.

This is an open access article licensed under the terms of the Creative Commons Attribution Non-Commercial License (http://creativecommons.org/licenses/by$\mathrm{nc} / 3.0 /$ ), which permits unrestricted, non-commercial use, distribution and reproduction in any medium, provided the work is properly cited. 